\title{
CHANGES IN HEALTH SCIENCES STUDENTS' PERCEPTION OF OBSTETRIC VIOLENCE AFTER AN EDUCATIONAL INTERVENTION
}

\begin{abstract}
Background: Obstetric violence is a type of gender-based violence that is presented structurally. This type of violence has physical and psychological consequences for both the women who experience it and health professionals. The World Health Organization adds that health professionals need training to ensure that pregnant women are treated with compassion and dignity.

Objectives: The objective of the study was to evaluate health sciences students' perception of obstetric violence and to identify possible changes after an educational intervention.
\end{abstract}

Design: A pre-post quasi-experimental study was carried out between January and June 2019.

Settings and participants: Students of medicine and nursing from Jaume I University (Universitat Jaume I) (Spain).

Methods: An ad hoc scale comprising 33 items was designed to measure the students' perceptions. In addition, sociodemographic and control variables were collected. Descriptive analyses of the sample and the scale were carried out, and a bivariate analysis was performed.

Results: Of the students surveyed, $89.7 \%$ were women, and the majority were nursing students. Of the 33 items, 28 (84.84\%) showed statistically significant changes in the pre-post-intervention measurement. Twenty-five of the 33 items (75.75\%) showed a relationship with the sociodemographic variables of gender, field, course and ever having been pregnant. 
Conclusion: This study shows the change in health sciences students' perceptions of obstetric violence after an educational intervention. In addition, the normalization of this type of violence was observed with the progression of training and with personal obstetric experience.

Keywords: Obstetric Violence; Perception

\section{Introduction}

The World Health Organization (WHO) states that "all women have the right to receive the highest level of health care, which includes the right to dignified and respectful care in pregnancy and childbirth, and the right not to suffer violence or discrimination" (WHO, 2014). In 1985, the European regional office of the WHO, the Pan American Health Organization and the regional office of the WHO for the Americas, at a conference on appropriate technology for childbirth, created a series of consensus recommendations among obstetricians, paediatricians, midwives, psychologists, epidemiologists, mothers and other professionals. The result was the "Declaration of Fortaleza" (World Health Organization, 1985), which the WHO considers applicable to all perinatal services throughout the world.

\section{Background}

Some definitions of obstetric violence (OV) exist. Specifically, the "Organic Law on the Right of Women to a Life Free of Violence", published in March 2007 in Venezuela, defines this term as "...the appropriation of the body and reproductive processes of women by health personnel, which is expressed as dehumanized treatment, an abuse of medication, and the conversion of natural processes into pathological ones, bringing with it loss of autonomy and the 
ability to decide freely about their bodies and sexuality, negatively impacting the quality of life of women" (Diaz-Tello, 2016; Pérez D'gregorio, 2010).

The WHO warns that an increasing number of studies on the experiences of women during pregnancy and, in particular, during childbirth present an alarming scenario, indicating that many women around the world experience disrespectful, offensive or negligent treatment during labour (WHO, 2014). In addition, it describes the practices that make OV visible: disrespectful and offensive treatment during childbirth, physical abuse, profound humiliation and verbal abuse, medical procedures performed without consent or under coercion (including sterilization), lack of confidentiality, failure to obtain the complete informed consent, refusal to administer analgesics, flagrant violations of privacy, refusal of admission to a health centre, negligence towards women during childbirth and the retention of women and new-borns in health centres due to their inability to pay (WHO, 2014), among others.

Concerning this type of violence, several conjectures have been raised regarding possible variables that favour its social stratification (Castro and Frías, 2019): lower socioeconomic level (Brandão et al., 2018; Santiago et al., 2018); youth, race, poor economic status and women's ignorance of their rights (Perera et al., 2018); or having dark skin (Grilo Diniz et al., 2018). However, an analysis of the main reasons and places of occurrence of this type of violence can reveal that $\mathrm{OV}$ is a type of structural violence.

The main reason for OV is gender bias, in which women's right to choose is nullified and replaced (Jardim and Modena, 2018). Regarding sites of occurrence, OV occurs throughout the world. Evidence shows that it exists in countries such as Mexico (Castro and Savage, 2019; Castro and Frías, 2019; 
Santiago et al., 2018), Venezuela, Argentina, Brazil (Ishola et al., 2017; Jardim and Modena, 2018), India (Bhattacharya and Sundari Ravindran, 2018), Tanzania (Mselle et al., 2018), the Czech Republic (Begley et al., 2018), Ecuador (Brandão et al., 2018), Italy (Castro and Frías, 2019; Ravaldi et al., 2018; Scambia et al., 2018), the United States (Perera et al., 2018), and Nigeria (Ishola et al., 2017), among other places. The structural nature of OV makes the health professional who exercises it unaware of it and even normalizes this practice (Borges, 2018).

Practices characterized by OV have physical and psychological consequences for both the women who experience them and the health professionals who practise or witness them. Women have shown how their physical, sexual and psychological health has been negatively affected (Chattopadhyay et al., 2018), and a very meaningful experience in their lives has been transformed into a violent and negative one (Borges, 2018; McGarry et al., 2017). On the other hand, the literature suggests that personnel who witness this type of violence during childbirth may suffer from compassion fatigue or secondary traumatic stress in response to observing the traumas the woman experiences first-hand (Sadler et al., 2016). The WHO states that it is necessary to generate data related to respectful and disrespectful care practices, responsibility systems and valuable professional support, adding that health professionals need support and training to ensure that pregnant women are treated with compassion and dignity (WHO, 2014).

Therefore, the main objective of this study was to evaluate the perceptions of health sciences students at Jaume I University (Universitat Jaume I) (Spain) 
have regarding OV and to detect possible changes in these perceptions after an educational intervention.

\section{Methods}

\section{Design and sample}

A pre-post quasi-experimental study was conducted among health sciences students at Jaume I University (Universitat Jaume I) (Spain) between January and June 2019.

A sample size calculation was performed using the GRANMO programme, which determined that a sample of 99 subjects was sufficient. The values considered for the calculation of the sample size included a confidence interval of $95 \%$, highlighting an initial proportion of events of 0.1 percentage points and a loss to follow-up of $20 \%$.

\section{Variables and instruments}

The sociodemographic variables that were considered were age, gender, field (medicine, nursing), course, health experience in gynaecology and obstetrics services (yes, no), duration of experience (less than 1 year, between 1 and 4 years, more than 4 years), having been present at a birth (yes, no), duration of experience being present at births (less than 1 year, between 1 and 4 years, more than 4 years), personal experience with pregnancies and births (yes, no), time since pregnancy and birth (less than 1 year, between 1 and 4 years, more than 4 years).

The perception of OV was measured with an ad hoc questionnaire composed of 33 items that referred to OV practices and were divided into 4 key moments 
(before delivery, during delivery, in case of caesarean section and after delivery). These items were measured using a 5-point Likert scale (1 strongly disagree - 5 strongly agree). The questionnaire was developed by a group of 3 experts and was based on the Guía de Práctica Clínica de Atención al Parto Normal (Clinical Practice of Normal Birth Care Guide) (Ministerio de Sanidad, 2014). The internal consistency of the scale, measured with Cronbach's alpha, was 0.922 for the pre-intervention measurement and 0.975 for the postintervention measurement.

The intervention consisted of an 8-hour seminar. This activity was composed of a theatrical performance on OV in the delivery room performed by "The Other Part of the Theatre" (1 hour); a master class on legal aspects presented by a lawyer specializing in health law (2 hours); a round table composed of professionals from the different fields, who contributed their experiences (4 hours); and another round table in which four volunteer mothers narrated their experiences of childbirth ( 1 hour). The session with the theatrical performance and the master class on legal issues was conducted on 03/07/2019. The round tables of the professionals and the mothers was held at a second session on 03/12/2019.

\section{Data collection}

Fieldwork was conducted in March 2019 after the launch of a seminar related to OV, in which students voluntarily enrolled. Data were collected through a selfcompleted survey administered before the students entered the seminar on $03 / 07 / 2019$ and after the activity on 03/12/2019. This survey was accompanied 
by an explanation of the study objective and an explanation of its voluntary and anonymous nature.

\section{Analysis}

A descriptive analysis of the data was performed considering means, standard deviations and $95 \%$ confidence intervals for the quantitative variables and the distribution of frequencies and percentages were taken into account for the qualitative variables. For the bivariate analysis, applicability was determined using parametric tests, the Kolmogorov-Smirnov normality test and Levene test for the homogeneity of variances. After these conditions were confirmed as acceptable, Student's T test was applied for paired data, with the intention of detecting the effect of the change in the different measurements, and the Mann Whitney $U$ and Kruskal Wallis tests were used to determine the relationships of the sociodemographic variables and perceptions of OV with the responses on the pre-intervention measurement. The analysis was carried out with the statistical package Statistical Package for Social Sciences (SPSS), version 21. A statistical significance level of $p<0.05$ was established.

\section{Ethical considerations}

The study was approved by the management of the Jaume I University (Universitat Jaume I) Nursing Research Group. The intervention was approved by the directorate of the Nursing Department and the dean of the School of Health Sciences of Jaume I University (Universitat Jaume I). Before data collection, the students received information about the objectives of the study as well as its methodology and the voluntary and anonymous nature of participation. The data collection tool did not include any personal data that 
could compromise the identity of the participants. The project was designed in accordance with the December 5 Protection of Personal Data and Guarantee of Digital Rights Organic Law 03/2018. In addition, the principles of the Declaration of Helsinki (charity, nonmaleficence, autonomy and justice) were respected. To respect the anonymity of the data and to match the first and second measurements, an ID was created consisting of the last two digits of the student's cell phone number, the last two digits of his or her National ID and his or her initials.

\section{Results}

A total of 107 questionnaires were collected. The mean age of the students was 22.5 years $( \pm 5.87)$. Women represented $89.7 \%$ of the sample $(n=96)$. A total of $86.9 \%(n=93)$ of the students belonged to the degree programme in nursing, and $28 \%(n=30)$ of the students had completed clinical practice in the gynaecology and obstetrics departments. Of the sample, $20.6 \%(n=22)$ had been present at a birth; only $4.7 \%(n=5)$ had been pregnant, and $2.8 \%(n=3)$ had given birth (Table 1).

Tabla 1. Variables sociodemográficas y de control de la muestra.

\begin{tabular}{|c|c|c|c|c|c|c|}
\hline \multirow[t]{2}{*}{ Variable } & \multicolumn{2}{|c|}{ Total } & \multicolumn{2}{|c|}{ Enfermería } & \multicolumn{2}{|c|}{ Medicina } \\
\hline & $\mathrm{n}$ & $\%$ & $\mathrm{n}$ & $\%$ & $\mathrm{n}$ & $\%$ \\
\hline \multicolumn{7}{|l|}{ Sexo } \\
\hline Masculino & 11 & 10.3 & 10 & 9.3 & 1 & 0.9 \\
\hline Femenino & 96 & 89.7 & 83 & 77.6 & 13 & 12.1 \\
\hline \multicolumn{7}{|l|}{ Curso } \\
\hline Primero & 10 & 9.3 & 8 & 7.5 & 2 & 1.9 \\
\hline Segundo & 46 & 43.0 & 40 & 37.4 & 6 & 5.6 \\
\hline Tercero & 10 & 9.3 & 7 & 6.5 & 3 & 2.8 \\
\hline Cuarto & 40 & 37.4 & 37 & 34.6 & 3 & 2.8 \\
\hline \multicolumn{7}{|c|}{ Experiencia en el servicio de ginecología y obstetricia } \\
\hline Sí & 30 & 28.0 & 25 & 23.5 & 5 & 4.7 \\
\hline No & 77 & 72.0 & 68 & 63.6 & 9 & 8.4 \\
\hline \multicolumn{7}{|c|}{ Presencia de algún parto } \\
\hline Sí & 22 & 20.6 & 20 & 18.7 & 2 & 1.9 \\
\hline
\end{tabular}


Embarazo propio

\begin{tabular}{llll}
\hline Variable & $\begin{array}{l}\text { Medida pre- } \\
\text { intervención }\end{array}$ & $\begin{array}{c}\text { Medida post- } \\
\text { intervención }\end{array}$ & p-valor \\
\cline { 2 - 4 }
\end{tabular}

\begin{tabular}{lcccccc} 
Sí & 5 & 4.7 & 5 & 4.7 & - & - \\
No & 102 & 95.3 & 88 & 82.2 & 14 & 13.1 \\
Tiempo desde el propio embarazo & & & & & & \\
Menos de un año & - & - & - & - & - & - \\
Entre 1 y 4 años & 2 & 1.9 & 2 & 1.9 & - & - \\
Más de 4 años & 3 & 2.8 & 3 & 2.8 & - & - \\
$\begin{array}{l}\text { Parto propio } \\
\text { Sí }\end{array}$ & 3 & 2.8 & 3 & 2.8 & - & - \\
No & 104 & 97.2 & 90 & 84.1 & 14 & 13.1 \\
Tiempo desde el propio parto & & & & & & - \\
Menos de un año & - & - & - & - & - & - \\
Entre 1 y 4 años & - & - & - & - & - & - \\
Más de 4 años & 3 & 2.8 & 3 & 2.8 & - & - \\
\hline
\end{tabular}

The Kolmogorov-Smirnov test $(p<0.01)$ and the Levene test $(p<0.05)$ verified the appropriateness of the Student's t-test for paired data. Table 2 shows the descriptive analysis of the variables for the pre- and post-intervention measurements and the bivariate analysis. All analyses of the OV perception scale by paired data showed statistically significant differences between the pre- and post-intervention measures, except on the items related to performing a pelvic examination without consent $(p=0.368)$; not preserving the privacy of the woman $(p=0.389)$; not considering the woman's decision $(p=0.086)$; taking pictures without permission ( $p=0.379)$; saying "Stop complaining, it is not that bad" $(p=0.181)$; Separating the mother and new-born $(p=1.00)$; and giving formula to the baby without the mother's consent $(p=0.320)$.

Tabla 2. Resultados de la percepción de los estudiantes en relación a la Violencia Obstétrica. 


\begin{tabular}{|c|c|c|c|c|c|}
\hline \multirow{2}{*}{\multicolumn{6}{|c|}{ Canalizar vía intravenosa }} \\
\hline & & & & & \\
\hline Nada de acuerdo & 44 & 41.9 & 2 & 2.0 & \multirow{5}{*}{$<0.01$} \\
\hline Algo de acuerdo & 20 & 19.0 & 7 & 7.1 & \\
\hline Ni de acuerdo ni en desacuerdo & 25 & 23.8 & 16 & 16.3 & \\
\hline Bastante de acuerdo & 13 & 12.4 & 37 & 37.8 & \\
\hline Muy de acuerdo & 3 & 2.9 & 36 & 36.7 & \\
\hline \multicolumn{6}{|l|}{ Dirigir en la posición } \\
\hline Nada de acuerdo & 52 & 49.5 & 8 & 8.2 & \multirow{5}{*}{$<0.01$} \\
\hline Algo de acuerdo & 19 & 18.1 & 3 & 3.1 & \\
\hline Ni de acuerdo ni en desacuerdo & 10 & 9.5 & 16 & 16.3 & \\
\hline Bastante de acuerdo & 17 & 16.2 & 28 & 28.6 & \\
\hline Muy de acuerdo & 7 & 6.7 & 43 & 43.9 & \\
\hline \multicolumn{5}{|c|}{ Acelerar el proceso de parto artificialmente } & \multirow{6}{*}{$<0.01$} \\
\hline Nada de acuerdo & 11 & 10.5 & 2 & 2.0 & \\
\hline Algo de acuerdo & 18 & 17.1 & 2 & 2.0 & \\
\hline Ni de acuerdo ni en desacuerdo & 26 & 24.8 & 4 & 4.1 & \\
\hline Bastante de acuerdo & 24 & 22.9 & 16 & 16.3 & \\
\hline Muy de acuerdo & 26 & 24.8 & 74 & 75.5 & \\
\hline \multicolumn{6}{|l|}{ Administrar enema de rutina } \\
\hline Nada de acuerdo & 18 & 17.0 & 2 & 2.0 & \multirow{5}{*}{$<0.01$} \\
\hline Algo de acuerdo & 16 & 15.1 & 2 & 2.0 & \\
\hline Ni de acuerdo ni en desacuerdo & 30 & 28.3 & 1 & 1.0 & \\
\hline Bastante de acuerdo & 19 & 17.9 & 18 & 18.4 & \\
\hline Muy de acuerdo & 23 & 21.7 & 75 & 76.5 & \\
\hline \multicolumn{6}{|l|}{ Amniorexis de rutina } \\
\hline Nada de acuerdo & 3 & 2.8 & 2 & 2.0 & \multirow{5}{*}{$<0.01$} \\
\hline Algo de acuerdo & 1 & 0.9 & 2 & 2.0 & \\
\hline Ni de acuerdo ni en desacuerdo & 21 & 19.8 & - & - & \\
\hline Bastante de acuerdo & 40 & 37.7 & 6 & 6.1 & \\
\hline Muy de acuerdo & 41 & 38.7 & 88 & 89.8 & \\
\hline \multicolumn{6}{|l|}{ Rasurado genital de rutina } \\
\hline Nada de acuerdo & 16 & 15.2 & 1 & 1.0 & \multirow{5}{*}{$<0.01$} \\
\hline Algo de acuerdo & 15 & 14.3 & 1 & 1.0 & \\
\hline Ni de acuerdo ni en desacuerdo & 24 & 22.9 & 7 & 7.1 & \\
\hline Bastante de acuerdo & 17 & 16.2 & 21 & 21.4 & \\
\hline Muy de acuerdo & 33 & 31.4 & 68 & 69.4 & \\
\hline \multicolumn{6}{|l|}{ Inmovilizar a la mujer } \\
\hline Nada de acuerdo & 2 & 1.9 & 2 & 2.0 & \multirow{5}{*}{$<0.01$} \\
\hline Algo de acuerdo & 3 & 2.8 & 1 & 1.0 & \\
\hline $\mathrm{Ni}$ de acuerdo ni en desacuerdo & 4 & 3.8 & - & - & \\
\hline Bastante de acuerdo & 29 & 27.4 & 3 & 3.1 & \\
\hline Muy de acuerdo & 68 & 64.2 & 92 & 93.9 & \\
\hline \multicolumn{6}{|l|}{ Tacto vaginal sin consentimiento } \\
\hline Nada de acuerdo & 2 & 1.9 & 2 & 2.0 & \multirow{3}{*}{0.368} \\
\hline Algo de acuerdo & 1 & 0.9 & 1 & 1.0 & \\
\hline Ni de acuerdo ni en desacuerdo & 2 & 1.9 & - & - & \\
\hline
\end{tabular}




\begin{tabular}{|c|c|c|c|c|c|}
\hline Bastante de acuerdo & 6 & 5.7 & 3 & 3.1 & \\
\hline Muy de acuerdo & 95 & 89.6 & 92 & 93.9 & \\
\hline \multicolumn{6}{|l|}{ No ofrecer medidas para el dolor } \\
\hline Nada de acuerdo & 2 & 1.9 & 3 & 3.1 & \multirow{5}{*}{$<0.01$} \\
\hline Algo de acuerdo & 2 & 1.9 & 3 & 3.1 & \\
\hline Ni de acuerdo ni en desacuerdo & 4 & 3.8 & - & - & \\
\hline Bastante de acuerdo & 32 & 30.2 & 9 & 9.2 & \\
\hline Muy de acuerdo & 66 & 62.3 & 83 & 84.7 & \\
\hline \multicolumn{6}{|l|}{ Incitar al uso de la epidural } \\
\hline Nada de acuerdo & 2 & 1.9 & 1 & 1.0 & \multirow{5}{*}{$<0.01$} \\
\hline Algo de acuerdo & 7 & 6.6 & 1 & 1.0 & \\
\hline $\mathrm{Ni}$ de acuerdo ni en desacuerdo & 22 & 20.8 & 5 & 5.1 & \\
\hline Bastante de acuerdo & 39 & 36.8 & 24 & 24.5 & \\
\hline Muy de acuerdo & 36 & 34.0 & 67 & 68.4 & \\
\hline \multicolumn{6}{|l|}{ No preservar la intimidad } \\
\hline Nada de acuerdo & 2 & 1.9 & 2 & 2.0 & \multirow{5}{*}{0.389} \\
\hline Algo de acuerdo & 1 & 0.9 & 1 & 1.0 & \\
\hline Ni de acuerdo ni en desacuerdo & 1 & 0.9 & - & - & \\
\hline Bastante de acuerdo & 9 & 8.5 & 6 & 6.4 & \\
\hline Muy de acuerdo & 93 & 87.7 & 89 & 90.8 & \\
\hline \multicolumn{6}{|c|}{ Convencer a la mujer de una cesárea para terminar el parto rápido y sin dolor } \\
\hline Nada de acuerdo & 2 & 1.9 & 2 & 2.0 & \multirow{5}{*}{$<0.01$} \\
\hline Algo de acuerdo & 5 & 4.7 & 2 & 2.0 & \\
\hline Ni de acuerdo ni en desacuerdo & 3 & 2.8 & - & - & \\
\hline Bastante de acuerdo & 27 & 25.5 & 5 & 5.1 & \\
\hline Muy de acuerdo & 69 & 65.1 & 89 & 90.8 & \\
\hline \multicolumn{6}{|c|}{ No considerar la decisión de la mujer } \\
\hline Nada de acuerdo & 1 & 0.9 & 2 & 2.0 & \multirow{5}{*}{0.086} \\
\hline Algo de acuerdo & 1 & 0.9 & - & - & \\
\hline Ni de acuerdo ni en desacuerdo & 2 & 1.9 & - & - & \\
\hline Bastante de acuerdo & 13 & 12.3 & 4 & 4.1 & \\
\hline Muy de acuerdo & 89 & 84.0 & 92 & 93.9 & \\
\hline \multicolumn{6}{|l|}{ Tomar imágenes sin permiso } \\
\hline Nada de acuerdo & 3 & 2.8 & 3 & 3.1 & \multirow{5}{*}{0.379} \\
\hline Algo de acuerdo & 1 & 0.9 & 1 & 1.0 & \\
\hline Ni de acuerdo ni en desacuerdo & - & - & - & - & \\
\hline Bastante de acuerdo & 6 & 5.7 & - & - & \\
\hline Muy de acuerdo & 96 & 90.6 & 94 & 95.9 & \\
\hline \multicolumn{6}{|l|}{ Posición de litotomía } \\
\hline Nada de acuerdo & 13 & 12.6 & 2 & 2.0 & \multirow{5}{*}{$<0.01$} \\
\hline Algo de acuerdo & 14 & 13.6 & 10 & 10.2 & \\
\hline Ni de acuerdo ni en desacuerdo & 31 & 30.1 & - & - & \\
\hline Bastante de acuerdo & 22 & 21.4 & 18 & 18.4 & \\
\hline Muy de acuerdo & 23 & 22.3 & 68 & 69.4 & \\
\hline \multicolumn{6}{|l|}{ Acompañamiento en el expulsivo } \\
\hline Nada de acuerdo & 29 & 27.6 & 8 & 8.4 & \\
\hline Algo de acuerdo & 16 & 15.2 & 4 & 4.2 & $<0.01$ \\
\hline
\end{tabular}




\begin{tabular}{|c|c|c|c|c|c|}
\hline Ni de acuerdo ni en desacuerdo & 17 & 16.2 & 12 & 12.6 & \\
\hline Bastante de acuerdo & 17 & 16.2 & 16 & 16.8 & \\
\hline Muy de acuerdo & 26 & 24.8 & 55 & 57.9 & \\
\hline \multicolumn{6}{|l|}{ Episiotomía de rutina } \\
\hline Nada de acuerdo & 1 & 1.0 & 2 & 2.0 & $<0.01$ \\
\hline Algo de acuerdo & 6 & 5.9 & 1 & 1.0 & \\
\hline Ni de acuerdo ni en desacuerdo & 13 & 12.7 & - & - & \\
\hline Bastante de acuerdo & 14 & 13.7 & 8 & 8.2 & \\
\hline Muy de acuerdo & 68 & 66.7 & 87 & 88.8 & \\
\hline \multicolumn{6}{|l|}{ Expresar: "No sabes empujar" } \\
\hline Nada de acuerdo & 3 & 2.9 & 2 & 2.0 & \\
\hline Algo de acuerdo & 2 & 1.9 & 1 & 1.0 & 0.05 \\
\hline Ni de acuerdo ni en desacuerdo & - & - & - & - & \\
\hline Bastante de acuerdo & 10 & 9.5 & 1 & 1.0 & \\
\hline Muy de acuerdo & 90 & 85.7 & 94 & 95.9 & \\
\hline \multicolumn{6}{|l|}{ Maniobra Kristeller } \\
\hline Nada de acuerdo & 1 & 1.0 & 2 & 2.0 & \\
\hline Algo de acuerdo & 2 & 1.9 & 1 & 1.0 & $<0.01$ \\
\hline Ni de acuerdo ni en desacuerdo & 11 & 10.6 & - & - & \\
\hline Bastante de acuerdo & 18 & 17.3 & 5 & 5.1 & \\
\hline Muy de acuerdo & 72 & 69.2 & 90 & 91.8 & \\
\hline \multicolumn{6}{|l|}{ Episiotomía sin anestesia } \\
\hline Nada de acuerdo & 4 & 4.0 & 2 & 2.0 & \\
\hline Algo de acuerdo & 2 & 2.0 & 1 & 1.0 & $<0.01$ \\
\hline Ni de acuerdo ni en desacuerdo & 10 & 9.9 & 1 & 1.0 & \\
\hline Bastante de acuerdo & 18 & 17.8 & - & - & \\
\hline Muy de acuerdo & 67 & 66.3 & 83 & 84.7 & \\
\hline \multicolumn{6}{|l|}{ Prohibir comer y beber } \\
\hline Nada de acuerdo & 8 & 7.6 & 2 & 2.0 & \\
\hline Algo de acuerdo & 11 & 10.5 & 5 & 5.1 & $<0.01$ \\
\hline Ni de acuerdo ni en desacuerdo & 29 & 27.6 & - & - & \\
\hline Bastante de acuerdo & 33 & 31.4 & 19 & 19.4 & \\
\hline Muy de acuerdo & 24 & 22.9 & 72 & 73.5 & \\
\hline \multicolumn{6}{|c|}{ No proporcionar abrigo/calefacción durante el parto } \\
\hline Nada de acuerdo & 1 & 1.0 & 4 & 4.1 & \\
\hline Algo de acuerdo & 4 & 3.8 & 1 & 1.0 & 0.01 \\
\hline Ni de acuerdo ni en desacuerdo & 7 & 6.7 & - & - & \\
\hline Bastante de acuerdo & 30 & 28.6 & 11 & 11.3 & \\
\hline Muy de acuerdo & 63 & 60.0 & 81 & 83.5 & \\
\hline \multicolumn{6}{|c|}{ Expresar: "Deja de quejarte, que tampoco es para tanto" } \\
\hline Nada de acuerdo & 3 & 2.9 & 1 & 1.0 & \\
\hline Algo de acuerdo & 1 & 1.0 & 1 & 1.0 & 0.181 \\
\hline Ni de acuerdo ni en desacuerdo & - & - & 1 & 1.0 & \\
\hline Bastante de acuerdo & 9 & 8.6 & 15 & 15.3 & \\
\hline Muy de acuerdo & 92 & 87.6 & 80 & 81.6 & \\
\hline \multicolumn{6}{|l|}{ Impedir que la mujer grite } \\
\hline Nada de acuerdo & 2 & 1.9 & 3 & 3.1 & \\
\hline
\end{tabular}




\begin{tabular}{|c|c|c|c|c|c|}
\hline Algo de acuerdo & 2 & 1.9 & 2 & 2.0 & 0.012 \\
\hline Ni de acuerdo ni en desacuerdo & 10 & 9.6 & - & - & \\
\hline Bastante de acuerdo & 17 & 16.3 & 6 & 6.1 & \\
\hline Muy de acuerdo & 73 & 70.2 & 87 & 88.8 & \\
\hline \multicolumn{6}{|c|}{ Realizar cesárea por lentitud al dilatar } \\
\hline Nada de acuerdo & 5 & 4.8 & 1 & 1.0 & \\
\hline Algo de acuerdo & 6 & 5.7 & 1 & 1.0 & \\
\hline Ni de acuerdo ni en desacuerdo & 16 & 15.2 & 1 & 1.0 & $<0.01$ \\
\hline Bastante de acuerdo & 32 & 30.5 & 15 & 15.3 & \\
\hline Muy de acuerdo & 46 & 43.8 & 80 & 81.6 & \\
\hline \multicolumn{6}{|c|}{ Cesárea de urgencia sin consentimiento } \\
\hline Nada de acuerdo & 5 & 4.8 & 1 & 1.0 & \\
\hline Algo de acuerdo & 3 & 2.9 & 1 & 1.0 & $<0.01$ \\
\hline Ni de acuerdo ni en desacuerdo & 8 & 7.7 & 2 & 2.0 & \\
\hline Bastante de acuerdo & 20 & 19.2 & 12 & 12.2 & \\
\hline Muy de acuerdo & 68 & 65.4 & 82 & 83.7 & \\
\hline \multicolumn{6}{|c|}{ Impedir el acompañamiento en caso de instrumentación o cesárea } \\
\hline Nada de acuerdo & 1 & 1.0 & 2 & 2.0 & \\
\hline Algo de acuerdo & 3 & 2.9 & 1 & 1.0 & $<0.01$ \\
\hline Ni de acuerdo ni en desacuerdo & 9 & 8.6 & 1 & 1.0 & \\
\hline Bastante de acuerdo & 23 & 21.9 & 5 & 5.1 & \\
\hline Muy de acuerdo & 69 & 65.7 & 89 & 90.8 & \\
\hline \multicolumn{6}{|l|}{ Corte de cordón inmediato } \\
\hline Nada de acuerdo & 10 & 9.6 & 2 & 2.0 & \\
\hline Algo de acuerdo & 15 & 14.4 & 2 & 2.0 & 0.018 \\
\hline Ni de acuerdo ni en desacuerdo & 20 & 19.2 & 10 & 10.2 & \\
\hline Bastante de acuerdo & 30 & 28.8 & 30 & 30.6 & \\
\hline Muy de acuerdo & 29 & 27.9 & 54 & 55.1 & \\
\hline \multicolumn{6}{|l|}{ Suturar sin anestesia un desgarro } \\
\hline Nada de acuerdo & 2 & 1.9 & 3 & 3.1 & \\
\hline Algo de acuerdo & 4 & 3.8 & 1 & 1.0 & $<0.01$ \\
\hline Ni de acuerdo ni en desacuerdo & 7 & 6.7 & 1 & 1.0 & \\
\hline Bastante de acuerdo & 29 & 27.9 & 8 & 8.2 & \\
\hline Muy de acuerdo & 62 & 59.6 & 85 & 86.7 & \\
\hline \multicolumn{6}{|l|}{ Separación madre-recién nacido } \\
\hline Nada de acuerdo & 2 & 1.9 & 2 & 2.0 & \\
\hline Algo de acuerdo & - & - & 1 & 1.0 & 1.00 \\
\hline Ni de acuerdo ni en desacuerdo & - & - & - & - & \\
\hline Bastante de acuerdo & 3 & 2.9 & 3 & 3.1 & \\
\hline Muy de acuerdo & 100 & 95.2 & 92 & 93.9 & \\
\hline \multicolumn{6}{|c|}{ Piel con piel tras la revisión pediátrica } \\
\hline Nada de acuerdo & 13 & 12.4 & 2 & 2.0 & \\
\hline Algo de acuerdo & 5 & 4.8 & 1 & 1.0 & $<0.01$ \\
\hline Ni de acuerdo ni en desacuerdo & 17 & 16.2 & 3 & 3.1 & \\
\hline Bastante de acuerdo & 21 & 20.0 & 16 & 16.3 & \\
\hline Muy de acuerdo & 49 & 46.7 & 76 & 77.6 & \\
\hline
\end{tabular}

Llevar el bebé al nido 
Nada de acuerdo

Algo de acuerdo

7

Ni de acuerdo ni en

6

Bastante de acuerdo

24

6.7

2

2.1

Muy de acuerdo

1

1.0

$<0.01$

32

22.9

8.2

$\begin{array}{lll}34.6 & 14 & 14.4\end{array}$

Dar leche artificial sin consentimiento de la madre

Nada de acuerdo

3

30.5

74.2

Algo de acuerdo

$\mathrm{Ni}$ de acuerdo ni en desacuerdo

Bastante de acuerdo

2.9

72

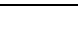

Muy de acuerdo

*T de Student para muestras relacionadas

Of the 33 items on the OV perception scale, only $24.24 \%(n=8)$ had no relationship with the sample's sociodemographic and control variables. The variables with the most statistically significant differences in relation to the OV perception scale were gender, course, and having been pregnant. Field (nursing vs. medicine) showed statistically significant differences on the items related to not offering measures for pain (nursing: $m=4.43, S D=0.85$, medicine: $\mathrm{m}=4.86, \mathrm{SD}=0.36, \mathrm{p}=0.047$ ); performing the Kristeller manoeuvre (nursing: $m=4.60, S D=0.75 ;$ medicine: $m=3.92, S D=1.17, p=<0.01$ ); performing an episiotomy without anaesthesia (nursing: $m=4.46, S D=1.01$; medicine: $m=3.89, S D=1.05 ; p=0.033)$; allowing skin-to-skin contact after the paediatric examination (nursing: $\mathrm{m}=3.93, \mathrm{SD}=1.35$; medicine: $\mathrm{m}=3.15$, $\mathrm{SD}=1.52, \mathrm{p}=0.049$ ) and taking the new-born to the nursery (nursing: $\mathrm{m}=$ 3.85, $\mathrm{SD}=1.12 ;$ medicine: $\mathrm{m}=3.15, \mathrm{SD}=1.14, \mathrm{p}=0.025)$ (Table 3 ). 


\section{Tabla 3. Resultados cruzados entre la percepción y las variables sociodemográficas}

\begin{tabular}{|c|c|c|c|c|c|c|c|}
\hline Variable & $\operatorname{Sexo}^{\mathrm{a}}$ & Disciplina $^{b}$ & Curso $^{c}$ & $\begin{array}{l}\text { Prácticas en } \\
\text { servicio de } \\
\text { ginecología- } \\
\text { obstetricia }^{d}\end{array}$ & $\begin{array}{l}\text { Presenciar } \\
\text { algún parto }^{\mathrm{e}}\end{array}$ & $\begin{array}{c}\text { Embarazo } \\
\text { propio }^{f}\end{array}$ & $\begin{array}{c}\text { Parto } \\
\text { propiog }^{\text {g }}\end{array}$ \\
\hline Canalizar vía intravenosa & & & $<0.01$ & $<0.01$ & & & $<0.01$ \\
\hline Dirigir en la posición & & & 0.01 & 0.01 & & & \\
\hline Administrar enema de rutina & 0.025 & & & & & 0.05 & $<0.01$ \\
\hline Amniorexis de rutina & & & & & & $<0.01$ & \\
\hline Rasurado genital de rutina & & & & & & $<0.01$ & $<0.01$ \\
\hline Tacto vaginal sin consentimiento & 0.073 & & $<0.01$ & & & $<0.01$ & $<0.01$ \\
\hline No ofrecer medidas para el dolor & & & $<0.01$ & & & 0.04 & $<0.01$ \\
\hline Incitar al uso de la epidural & 0.029 & & & & & $<0.01$ & $<0.01$ \\
\hline No preservar la intimidad & $<0.01$ & & & & & $<0.01$ & $<0.01$ \\
\hline No considerar la decisión de la mujer & & & 0.03 & 0.043 & & & $<0.01$ \\
\hline Tomar imágenes sin permiso & $<0.01$ & & $<0.01$ & & & 0.047 & $<0.01$ \\
\hline Episiotomía de rutina & $<0.01$ & & 0.031 & & & $<0.01$ & $<0.01$ \\
\hline Expresar: "No sabes empujar" & 0.048 & $<0.01$ & & & 0.012 & $<0.01$ & $<0.01$ \\
\hline Maniobra Kristeller & & $<0.01$ & 0.057 & & & & \\
\hline Episiotomía sin anestesia & $<0.01$ & 0.061 & 0.013 & & & 0.048 & \\
\hline Prohibir comer y beber & $<0.01$ & & & & & & 0.025 \\
\hline Expresar: "Deja de quejarte, que tampoco es para tanto" & & & $<0.01$ & & & $<0.01$ & $<0.01$ \\
\hline Impedir que la mujer grite & 0.056 & & & & & 0.032 & $<0.01$ \\
\hline Realizar cesárea por lentitud al dilatar & & & & & & $<0.01$ & 0.015 \\
\hline Cesárea de urgencia sin consentimiento & & & 0.030 & 0.043 & & & $<0.01$ \\
\hline Impedir el acompañamiento en caso de instrumentación o cesárea & 0.012 & 0.021 & & & & & $<0.01$ \\
\hline Suturar sin anestesia un desgarro & $<0.01$ & & & & & 0.039 & $<0.01$ \\
\hline Separación madre-recién nacido & 0.49 & & & & & & $<0.01$ \\
\hline Piel con piel tras la revisión pediátrica & & & $<0.01$ & 0.048 & 0.036 & & \\
\hline Dar leche artificial sin consentimiento de la madre & & & $<0.01$ & & & & $<0.01$ \\
\hline
\end{tabular}

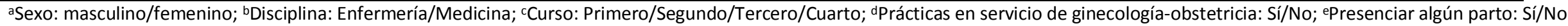
fEmbarazo propio: Sí/No; sParto propio: Sí/No 
The completion of rotations in obstetrics-gynaecology was statistically significant related to the variables on the OV perception scale: directing the position of the woman in labour (yes: $m=2.57, S D=1.33$; no: $m=1.95, S D=$ 1.33; $p=0.025$ ); performing genital shaving (yes: $m=3.80, S D=1.29 ; n o: ~ m=$ $3.16, S D=1.46 ; p=0.041)$ and convincing the woman to undergo a caesarean section to end the labour quickly and without pain (yes: $m=4.70, S D=0.79$; no: $m=4.38, S D=0.93 ; p=0.021$ ). Having been present at a childbirth was statistically significantly related to the following variable on the OV perception scale: Saying "you do not know how to push" (yes: $\mathrm{m}=4.45, \mathrm{SD}=1.01$; no: $\mathrm{m}$ $=4.84, \mathrm{SD}=0.65 ; \mathrm{p}=<0.01$ ).

Table 4 shows the descriptive and comparative results for the control variables with respect to the items on the OV perception scale. Having given birth was statistically significantly related to the following items on the scale: performing routine genital shaving (yes: $m=1.00, S D=0.00 ; n o: m=3.41, S D=1.40, p=$ $<0.01$ ), performing routine episiotomy (yes: $\mathrm{m}=2.67, \mathrm{SD}=0.57$; no: $\mathrm{m}=4.44$, $S D=0.95 ; p=<0.01$ ); saying "you do not know how to push" (yes: $m=2.33$, $S D=1.15 ;$ no: $m=4.82, S D=0.62 ; p=<0.01$ ); performing a caesarean section due to slow dilation (yes: $m=2.00, S D=1.00 ; n o: ~ m=4.09, S D=1.07 ; p=$ $<0.01$ ) and not allowing company in cases of instrumentation or caesarean section (yes: $m=3.33, S D=0.57 ;$ no: $m=4.09, S D=1.07 ; p=0.02$ ). Having been pregnant was statistically significantly related to the following items: performing routine genital shaving (yes: $\mathrm{m}=1.80 ; \mathrm{SD}=1.78 ; \mathrm{no}: \mathrm{m}=3.42$, $\mathrm{SD}$ $=1.38 ; p=0.028$ ); performing a pelvic exam without consent (yes: $\mathrm{m}=4.20$, $S D=1.30 ; n o: m=4.83, S D=0.64, p=0.026)$; encouraging the use of an 
epidural (yes: $m=4.40, S D=0.54$; no: $m=4.81, S D=0.68, p=<0.01$ ); not considering the woman's decision (yes: $m=4.40, S D=0.54 ;$ no: $m=4.79$, SD $=0.62 ; p=0.01$ ); taking pictures without permission (yes: $m=4.00, S D=1.73$; no: $m=4.85, S D=0.62 ; p=0.015$ ); performing routine episiotomy (yes: $m=$ $3.00, S D=0.70 ;$ no: $m=4.46, S D=0.94 ; p=<0.01)$; saying "you do not know how to push" (yes: $m=3.40, S D=1.67 ;$ no: $m=4.82, S D=0.62 ; p=<0.01$ ); saying "stop complaining, it is not that bad" (yes: $\mathrm{m}=3.80, \mathrm{SD}=1.78$; $\mathrm{no}$ : $\mathrm{m}=$ $4.83, \mathrm{SD}=0.62 ; \mathrm{p}=0.034) ;$ and performing a caesarean due to slow dilation (yes: $m=2.60 ; S D=1.51 ; n o: m=4.10, S D=1.05 ; p=0.022$ ).

\section{Discussion}

It is important to emphasize that students' participation in the proposed teaching activity was much greater than initially expected. The students seemed very motivated by the central theme, and their involvement was notable; therefore, the capture of individuals from the sample exceeded the calculated sample size. The high percentage of women in the sample (89.7\%) should be noted; it may have occurred because women were especially motivated by the issue or because female representation is increasing in the health sciences (BernalteMartí, 2015). It is noteworthy that students in different years of their programme were equitably represented in the seminar, although more second- and fourthyear students than students of other years were in attendance. The representation of medical students was low; some possible reasons for this low attendance may be low dissemination of the activity among these students or the possibility that nursing students feel more linked to this type of practice (Olza-Fernández and Ruiz-Berdún, 2015). Because our sample was young in 
relation to the mean age (32.58 years) for maternity in Spain according to data from the National Institute of Statistics (Instituto Nacional de Estadística INE)(«Edad Media a la Maternidad por orden del nacimiento según nacionalidad (española/extranjera) de la madre(1579)», s. f.), very few participants had a personal medical history of pregnancy or birth, although $30 \%$ of the sample had experience in gynaecology and obstetrics.

Regarding the comparison of the pre- and post-intervention measures by paired data, it is noteworthy that with the exception of a few items, all measures presented statistically significant differences. Even so, it is worth highlighting the low results found in the pre-intervention measurement. The Fortaleza document (World Health Organization, 1985) states that a family member chosen by the mother may accompany her during childbirth and throughout the postnatal period to promote her well-being; the new-born should stay with his/her mother whenever possible; immediate breastfeeding should be promoted; the dorsal position of the woman in lithotomy during dilation is not recommended; and the shaving of pubic hair, the administration of enemas, the systematic use of episiotomy and the early artificial rupture of membranes should not be performed as routine procedures. All these procedures were included in the questionnaire, and of them, the only one that was identified as violence preintervention was "giving formula without the mother's consent".

It should be noted that health science students should be trained in the latest available evidence (Aglen, 2016). Apparently, this does not occur in the field of obstetrics in Spain, highlighting the need for all women of child-bearing age to receive evidence-based care that is applied respectfully without neglecting the woman's opinions and preferences (Begley et al., 2018). Along the same lines, 
the Fédération Internationale de Gynécologie et d'Obstétrique (FIGO) adds that "Every woman and every baby should be protected from unnecessary interventions, practices and procedures that are not evidence-based, and any practices that are not respectful of their culture, bodily integrity, and dignity" (International Federation of Gynaecology and Obstetrics et al., 2014). Special focus falls on the Kristeller manoeuvre, which, despite being contraindicated, continues to be put into practice (Borges, 2018; Fritz et al., 2017; Rubashkin et al., 2019) and was not recognized by the students as OV.

It is evident that there is a close relationship between ideological representations of the female gender and the existence of OV. The cultural image of women as reproductive and submissive serves as a precedent for the domination, control and abuse they experience in relation to their bodies and sexuality. As a consequence, women are nullified, and their rights to choose are replaced (Jardim and Modena, 2018). These assertions are corroborated by the results obtained in this study; when the responses to the OV scale were compared by gender, a large number of variables presented statistically significant differences, and in all of them, the perception of OV was higher among females.

Another feature of this OV is that it is rooted in a system that stands in the way of optimal health outcomes (Castro and Savage, 2019); thus, it also has a structural nature (Bhattacharya and Sundari Ravindran, 2018). In this way, the researchers assumed two facts that were confirmed through this study: a) the normalization of this type of violence according to the student's year of study, i.e., a lower perception of OV among more advanced students and a relationship between perceptions of OV and having participated in obstetric 
practices during study; and b) the normalization of this type of obstetric practices in relation to the participant's personal experience with pregnancies and births (a decreased perception of OV after having been pregnant or given birth). A larger study is necessary to determine the degree of normalization and the normalization process; however, given these preliminary data, it is essential to change the training of health personnel, who should have a solid foundation in ethics and gender and human rights because emotional factors or burnout may be among the reasons for practising OV (Olza Fernández, 2013). The strain on health personnel is so high that many professionals have to abandon their job and even their profession (Beck and Gable, 2012). Therefore, public policies must direct attention towards humane and respectful treatment that is based on and supported by the latest available evidence. However, in order for this to happen, health personnel who work with pregnant women must abandon the traditional hierarchy and structure in which medical supervision implies a subordination of women's bodies and sexuality. This fact is further aggravated when all attention is paid to techniques, and the value of how people are treated is lost (Grilo Diniz et al., 2018; Mselle et al., 2018). Education that promotes respect and informs and raises awareness among future professionals, along with policies, guides, protocols and education, will eradicate OV (Brandão et al., 2018; Diaz-Tello, 2016; Grilo Diniz et al., 2018; Mselle et al., 2018; Sen et al., 2018). Education is a fundamental aspect for ending the normalization of OV in society; it approaches the problem from the root and will evolve until the rights of women are respected.

\section{Conclusions}


According to the results of this study, health sciences students integrate the normalizations of OV during their studies. A formative activity aimed at making this type of violence visible and reflecting on OV helps to create awareness among students, making it possible for them to notice this type of violence and be able to identify it. It is noteworthy that from the beginning, the women in the study have perceived all the points raised on the OV scale as having higher OV; additionally, OV becomes normalized as a result of being present at a delivery, the progression of training (depending on the course) and obstetric experience itself, including pregnancies and births.

\section{References}

Aglen, B., 2016. Pedagogical strategies to teach bachelor students evidencebased practice: A systematic review. Nurse Educ. Today 36, 255-263. https://doi.org/10.1016/j.nedt.2015.08.025

Beck, C.T., Gable, R.K., 2012. A Mixed Methods Study of Secondary Traumatic Stress in Labor and Delivery Nurses. JOGNN - J. Obstet. Gynecol. Neonatal Nurs. $\quad 41, \quad 747-760 . \quad$ https://doi.org/10.1111/j.1552$6909.2012 .01386 . x$

Begley, C., Sedlicka, N., Daly, D., 2018. Respectful and disrespectful care in the Czech Republic: An online survey. Reprod. Health 15, 198. https://doi.org/10.1186/s12978-018-0648-7

Bernalte-Martí, V., 2015. The minority of male in the nursing profession. Reflexions of their history, image and their evolution in Spain. Enfermería Glob. 14, 328-334.

Bhattacharya, S., Sundari Ravindran, T.K., 2018. Silent voices: Institutional 
disrespect and abuse during delivery among women of Varanasi district, northern India. BMC Pregnancy Childbirth 18, 338. https://doi.org/10.1186/s12884-018-1970-3

Borges, M.T., 2018. A Violent Birth: Reframing Coerced Procedures During Childbirth as Obstetric Violence. Duke Law J. 67, 827-862.

Brandão, T., Cañadas, S., Galvis, A., de los Ríos, M.M., Meijer, M., Falcon, K., 2018. Childbirth experiences related to obstetric violence in public health units in Quito, Ecuador. Int. J. Gynecol. Obstet. 143, 84-88. https://doi.org/10.1002/ijgo.12625

Castro, A., Savage, V., 2019. Obstetric Violence as Reproductive Governance in the Dominican Republic. Med. Anthropol. Cross Cult. Stud. Heal. Illn. 38, 123-136. https://doi.org/10.1080/01459740.2018.1512984

Castro, R., Frías, S.M., 2019. Obstetric Violence in Mexico: Results From a 2016 National Household Survey. Violence Against Women. https://doi.org/10.1177/1077801219836732

Chattopadhyay, S., Mishra, A., Jacob, S., 2018. 'Safe', yet violent? Women's experiences with obstetric violence during hospital births in rural Northeast $\begin{array}{llll}\text { India. } & \text { Cult. } & \text { Heal. } & \text { Sex. }\end{array}$ https://doi.org/10.1080/13691058.2017.1384572

Diaz-Tello, F., 2016. Invisible wounds: obstetric violence in the United States. Reprod. Health $\quad$ Matters 24, 56-64. https://doi.org/10.1016/j.rhm.2016.04.004

Edad Media a la Maternidad por orden del nacimiento según nacionalidad (española/extranjera) de la madre (1579) [WWW Document], s. f. URL http://www.ine.es/jaxiT3/Datos.htm?t=1579 (Accessed: 03.05.2019). 
Fritz, J., Walker, D.M., Cohen, S., Angeles, G., Lamadrid-Figueroa, H., 2017. Can a simulation-based training program impact the use of evidence based routine practices at birth? Results of a hospital-based cluster randomized trial in Mexico. PLoS One 12, e0172623. https://doi.org/10.1371/journal.pone.0172623

Grilo Diniz, C.S., Rattner, D., Lucas d'Oliveira, A.F.P., de Aguiar, J.M., Niy, D.Y., 2018. Disrespect and abuse in childbirth in Brazil: social activism, public policies and providers' training. Reprod. Health Matters 26, 19-35. https://doi.org/10.1080/09688080.2018.1502019

International Federation of Gynecology and Obstetrics, International Confideration of Midwives, White Ribbon Alliance, International Pediatric Association, World Health Organization, 2014. Mother-baby friendly birthing facilities. Int. J. Gynecol. Obstet. https://doi.org/10.1016/j.ijgo.2014.10.013

Ishola, F., Owolabi, O., Filippi, V., 2017. Disrespect and abuse of women during childbirth in Nigeria: A systematic review. PLoS One 12, e0174084. https://doi.org/10.1371/journal.pone.0174084

Jardim, D.M.B., Modena, C.M., 2018. Obstetric violence in the daily routine of care and its characteristics. Rev. Lat. Am. Enfermagem 26, e3069. https://doi.org/10.1590/1518-8345.2450.3069

McGarry, J., Hinsliff-Smith, K., Watts, K., McCloskey, P., Evans, C., 2017. Experiences and impact of mistreatment and obstetric violence on women during childbearing: A systematic review protocol. JBI Database Syst. Rev. Implement. Reports 15, 620-627. https://doi.org/10.11124/JBISRIR-2016002950 
Ministerio de Sanidad, 2014. Guía de Práctica Clínica sobre la Atención al Parto Normal, Internet. https://doi.org/http://www.guiasalud.es/GPC/GPC_472_Parto_Normal_Oste ba_compl.pdf

Mselle, L.T., Kohi, T.W., Dol, J., 2018. Barriers and facilitators to humanizing birth care in Tanzania: Findings from semi-structured interviews with midwives and obstetricians. Reprod. Health 15, 137. https://doi.org/10.1186/s12978-018-0583-7

Olza-Fernández, I., Ruiz-Berdún, D., 2015. Midwifes experiences regarding obstetric violence, en: Archives of Women's Mental Health. https://doi.org/10.1007/s00737-014-0488-6

Olza Fernández, I., 2013. PTSD and obstetric violence. Midwifery Today Int. Midwife Spring, 48-49.

Perera, D., Lund, R., Swahnberg, K., Schei, B., Infanti, J.J., Darj, E., Lukasse, M., Bjørngaard, J.H., Joshi, S.K., Rishal, P., Koju, R., Pun, K.D., Wijewardena, K., Muzrif, M.M., Campbell, J.C., 2018. «When helpers hurt»: Women's and midwives' stories of obstetric violence in state health institutions, Colombo district, Sri Lanka. BMC Pregnancy Childbirth 18, 211. https://doi.org/10.1186/s12884-018-1869-z

Pérez D'gregorio, R., 2010. Obstetric violence: A new legal term introduced in Venezuela. Int. J. Gynecol. Obstet. 111, 201-202. https://doi.org/10.1016/j.ijgo.2010.09.002

Ravaldi, C., Skoko, E., Battisti, A., Cericco, M., Vannacci, A., 2018. Sociodemographic characteristics of women participating to the LOVETHEM (Listening to Obstetric Violence Experiences THrough Enunciations 
and Measurement) investigation in Italy. Data $\mathrm{Br} .19,226-229$. https://doi.org/10.1016/j.dib.2018.04.146

Rubashkin, N., Torres, C., Escuriet, R., Dolores Ruiz-Berdún, M., 2019. “Just a little help": A qualitative inquiry into the persistent use of uterine fundal pressure in the second stage of labor in Spain. Birth. https://doi.org/10.1111/birt.12424

Sadler, M., Santos, M.J., Ruiz-Berdún, D., Rojas, G.L., Skoko, E., Gillen, P., Clausen, J.A., 2016. Moving beyond disrespect and abuse: addressing the structural dimensions of obstetric violence. Reprod. Health Matters 24, 4755. https://doi.org/10.1016/j.rhm.2016.04.002

Santiago, R.V., Monreal, L.A., Rojas Carmona, A., Domínguez, M.S., 2018. «lf we're here, it's only because we have no money.» discrimination and violence in Mexican maternity wards. BMC Pregnancy Childbirth 18, 244. https://doi.org/10.1186/s12884-018-1897-8

Scambia, G., Viora, E., Chiantera, A., Colacurci, N., Vicario, M., 2018. "Obstetric violence": Between misunderstanding and mystification. Eur. J. Obstet. Gynecol. Reprod. Biol. Sep, 331. https://doi.org/10.1016/j.ejogrb.2018.06.012

Sen, G., Reddy, B., Iyer, A., 2018. Beyond measurement: the drivers of disrespect and abuse in obstetric care. Reprod. Health Matters 26, 6-18. https://doi.org/10.1080/09688080.2018.1508173

World Health Organization, 2014. The prevention and elimination of disrespect and abuse during facility-based childbirth., World Health Organization. https://doi.org/WHO/RHR/14.23

World Health Organization, 1985. Appropriate technology for birth. Lancet 24, 
436-437. 